\title{
SPECTRAL GAPS FOR PERIODIC SCHRÖDINGER OPERATORS WITH STRONG MAGNETIC FIELDS
}

\author{
YURI A. KORDYUKOV
}

\begin{abstract}
We consider Schrödinger operators $H^{h}=(i h d+\mathbf{A})^{*}(i h d+\mathbf{A})$ with the periodic magnetic field $\mathbf{B}=d \mathbf{A}$ on covering spaces of compact manifolds. Under some assumptions on $\mathbf{B}$, we prove that there are arbitrarily large number of gaps in the spectrum of these operators in the semiclassical limit of strong magnetic field $h \rightarrow 0$.
\end{abstract}

\section{INTRODUCTION}

Let $(M, g)$ be a closed Riemannian oriented manifold of dimension $n \geq 2, \widetilde{M}$ be its universal cover and $\widetilde{g}$ be the lift of $g$ to $\widetilde{M}$ so that $\widetilde{g}$ is a $\Gamma$-invariant Riemannian metric on $\widetilde{M}$ where $\Gamma$ denotes the fundamental group of $M$ acting on $\widetilde{M}$ by the deck transformations. Let $\mathbf{B}$ be a real-valued $\Gamma$-invariant closed 2 -form on $\widetilde{M}$. We assume that $\mathbf{B}$ is exact. Choose a real-valued 1-form $\mathbf{A}$ on $\widetilde{M}$ such that $d \mathbf{A}=\mathbf{B}$. Physically we can think of $\mathbf{A}$ as the electromagnetic vector potential for a magnetic field $\mathbf{B}$.

We consider the magnetic Schrödinger operator $H_{h}$ given by

$$
H^{h}=(i h d+\mathbf{A})^{*}(i h d+\mathbf{A}),
$$

acting on the Hilbert space $\mathfrak{H}=L^{2}(\widetilde{M})$. Here $h>0$ is a semiclassical parameter, which is assumed to be small.

In local coordinates $X=\left(X_{1}, \ldots, X_{n}\right)$, we write the 1 -form $\mathbf{A}$ as

$$
\mathbf{A}=A_{1}(X) d X_{1}+\ldots+A_{n}(X) d X_{n},
$$

the matrix of the Riemannian metric $\widetilde{g}$ as $g(X)=\left(g_{j l}(X)\right)_{1 \leq j, l \leq n}$ and its inverse as $\left(g^{j l}(X)\right)_{1 \leq j, l \leq n}$. If $|g(X)|=\operatorname{det}(g(X))$, then the Schrödinger operator $H_{h}$ is given by

$$
H_{h}=\frac{1}{\sqrt{|g(X)|}} \sum_{1 \leq j, l \leq n}\left(i h \frac{\partial}{\partial X_{j}}+A_{j}(X)\right)\left[\sqrt{|g(X)|} g^{j l}(X)\left(i h \frac{\partial}{\partial X_{l}}+A_{l}(X)\right)\right] \text {. }
$$

For any $x \in \widetilde{M}$ denote by $B(x)$ the anti-symmetric linear operator on the tangent space $T_{x} \widetilde{M}$ associated with the 2 -form $\mathbf{B}$ :

$$
\widetilde{g}_{x}(B(x) u, v)=\mathbf{B}_{x}(u, v), \quad u, v \in T_{x} \widetilde{M} .
$$

The trace-norm $|B(x)|$ of $B(x)$ is given by the formula

$$
|B(x)|=\left[\operatorname{Tr}\left(B^{*}(x) \cdot B(x)\right)\right]^{1 / 2} .
$$


We will assume that there exists an integer $k>0$ such that, if $B\left(x_{0}\right)=0$ then

$$
C_{1}^{-1} d\left(x, x_{0}\right)^{k} \leq|B(x)| \leq C_{1} d\left(x, x_{0}\right)^{k}
$$

in some neighborhood of $x_{0}$ (here $d$ denotes the geodesic distance on $\widetilde{M}$ ). We assume that there exists at least one zero of $B$.

Theorem 1. Under current assumptions, there exists an increasing sequence $\left\{\lambda_{m}, m \in\right.$ $\mathbb{N}\}$, satisfying $\lambda_{m} \rightarrow \infty$ as $m \rightarrow \infty$, such that for any a and $b$, satisfying $\lambda_{m}<a<b<$ $\lambda_{m+1}$ with some $m$, the interval $\left[a h^{\frac{2 k+2}{k+2}}, b h^{\frac{2 k+2}{k+2}}\right]$ does not meet the spectrum of $H^{h}$ for any $h>0$ small enough. It follows that there exists arbitrarily large number of gaps in the spectrum of $H^{h}$ provided the coupling constant $h$ is sufficiently small.

Here the sequence $\left\{\lambda_{m}\right\}$ appears as the set of eigenvalues of a model operator $K^{h}$ associated to $H^{h}$. This operator is defined as a direct sum of principal parts of $H^{h}$ near the zeroes of $B$ in a fundamental domain (see Section 2 for a precise definition). It is a differential operator, which acts on the Hilbert space $\mathfrak{H}_{K}=L^{2}\left(\mathbb{R}^{n}\right)^{N}$ and has discrete spectrum (here $n$ is the dimension of $M$ and $N$ denotes the number of zeroes of $B$ that lie in a fundamental domain). Using a simple scaling and gauge invariance, it can be shown that the operator $K^{h}$ is unitarily equivalent to the operator $h^{\frac{2 k+2}{k+2}} K^{1}$. Therefore the operator $h^{-\frac{2 k+2}{k+2}} K^{h}$ has discrete spectrum independent of $h$. This fact explains the appearance of a scaling factor $h^{\frac{2 k+2}{k+2}}$ in Theorem 1.

There exist a few examples of periodic partial differential operators of the second order with spectral gaps (see, for instance, 1, 2, 3, 10, 11, 13, and a recent survey [12] and references therein). In particular, in [10] Hempel and Herbst studied magnetic Schrödinger operators

$$
H(\lambda \vec{a})=(-i \nabla-\lambda \vec{a}(x))^{2}
$$

in $L^{2}\left(\mathbb{R}^{n}\right)$, where $\vec{a} \in C^{1}\left(\mathbb{R}^{n} ; \mathbb{R}^{n}\right)$ is a vector potential and $\lambda \in \mathbb{R}$. Let $M=\left\{x \in \mathbb{R}^{n}\right.$ : $B(x)=0\}$, where $B=d \vec{a}$ is the magnetic field associated with $\vec{a}$, and $M_{\vec{a}}=\left\{x \in \mathbb{R}^{n}\right.$ : $\vec{a}(x)=0\}$. They proved that, if $B$ is periodic with respect to the lattice $\mathbb{Z}^{n}$, the set $M \backslash M_{\vec{a}}$ has measure zero, the interior of $M$ is non-empty and $M$ can be represented as $M=\cup_{j \in \mathbb{Z}^{n}} M_{j}$ (up to a set of measure zero) where the $M_{j}$ are pairwise disjoint compact sets with $M_{j}=M_{0}+j$, then the spectrum of the operator $H(\lambda \vec{a})$ has arbitrarily large number of gaps provided the coupling constant $\lambda$ is sufficiently large. The proof of this result is based on the fact that, as $\lambda \rightarrow \infty, H(\lambda \vec{a})$ converges in norm resolvent sense to the Dirichlet Laplacian $-\Delta_{M}$ on the closed set $M$. Since norm resolvent convergence implies convergence of spectra, we immediately obtain that, as $\lambda \rightarrow \infty$, the spectrum of $H(\lambda \vec{a})$ concentrates around the eigenvalues of $-\Delta_{M}$ and gaps opens up in the spectrum of $H(\lambda \vec{a})$.

On the other hand, Hempel and Herbst also proved in 10 that, if $M_{\vec{a}}$ has measure zero, then, as $\lambda \rightarrow \infty, H(\lambda \vec{a})$ converges in strong resolvent sense to the zero operator in $L^{2}\left(\mathbb{R}^{n}\right)$. So, in this case, their method to produce operators with spectral gaps does not work. 
In this paper, we consider a particular case when $M_{\vec{a}}$ has measure zero. More precisely, Theorem 1 states that if $M_{\vec{a}}$ has measure zero and the magnetic field has a regular behaviour near its zeroes, we still can produce examples of magnetic Schrödinger operators $H(\lambda \vec{a})$ with arbitrarily large number of gaps in their spectra. The proof of Theorem 1 is based on Theorem 2 below.

Recall that the magnetic Schrödinger operator $H^{h}$ commutes with a projective $(\Gamma, \sigma)$ action of the fundamental group $\Gamma$, where $\sigma$ is the multiplier or $U(1)$-valued 2-cocycle on $\Gamma$ defining this projective action.

Consider the reduced twisted group $C^{*}$-algebra $C_{r}^{*}(\Gamma, \bar{\sigma})$ of the group $\Gamma$. If $\mathcal{H}$ is a Hilbert space, then let $\mathcal{K}(\mathcal{H})$ denote the algebra of compact operators in $\mathcal{H}$, and $\mathcal{K}=$ $\mathcal{K}\left(\ell^{2}(\mathbb{N})\right)$, where $\mathbb{N}=\{1,2,3, \ldots\}$. Let $E^{h}(\lambda)=\chi_{(-\infty, \lambda]}\left(H^{h}\right)$ and $E^{0}(\lambda)=\chi_{(-\infty, \lambda]}\left(K^{h}\right)$ denote the spectral projections. One can define actions of the $C^{*}$-algebra $C_{r}^{*}(\Gamma, \bar{\sigma}) \otimes \mathcal{K}$ in the Hilbert spaces $\mathfrak{H}$ and $\ell^{2}(\Gamma) \otimes \mathfrak{H}_{K}$. It can be shown that $E(\lambda)$ and id $\otimes E^{0}(\lambda)$ are in $C_{r}^{*}(\Gamma, \bar{\sigma}) \otimes \mathcal{K}$.

Recall that two projections $P$ and $Q$ in a unital $*$-algebra $\mathcal{A}$ are said to be Murray-von Neumann equivalent if there is an element $V \in \mathcal{A}$ such that $P=V^{*} V$ and $Q=V V^{*}$.

Theorem 2. Assume that $\lambda \in \mathbb{R}$ does not coincide with $\lambda_{k}$ for any $k$. There exists a $(\Gamma, \sigma)$-equivariant isometry $U: \mathfrak{H} \rightarrow \ell^{2}(\Gamma) \otimes \mathfrak{H}_{K}$ and a constant $h_{0}>0$ such that for all $h \in\left(0, h_{0}\right)$, the spectral projections $U E\left(h^{\frac{2 k+2}{k+2}} \lambda\right) U^{*}$ and $\mathrm{id} \otimes E^{0}\left(h^{\frac{2 k+2}{k+2}} \lambda\right)$ are Murray-von Neumann equivalent in $C_{r}^{*}(\Gamma, \bar{\sigma}) \otimes \mathcal{K}\left(\mathfrak{H}_{K}\right)$.

The proofs of Theorems 1 and 2 are based on abstract operator-theoretic results on equivalence of projections and existence of spectral gaps proved in [14. These results were applied in [14] to prove existence of arbitrarily large number of gaps in the spectrum of periodic magnetic Schrödinger operators

$$
H_{\mu}=(i d+\mathbf{A})^{*}(i d+\mathbf{A})+\mu^{-2} V(x)
$$

on the universal covering $\widetilde{M}$ of a compact manifold $M$ in the limit of the strong electric field $(\mu \rightarrow 0)$, where $\mathbf{B}=d \mathbf{A}$ is a $\Gamma$-invariant closed 2 -form on $\widetilde{M}$ and $V \geq 0$ is a $\Gamma$-invariant Morse potential (see also [15]).

The another important results, which we use in construction of the model operator and in the proof of Theorem 2, are connected with the study of Schrödinger operators with magnetic wells and were obtained by Hellfer and Mohamed (=Morame) in ([5], see also [6, 7, 8] for further developments).

The paper is organized as follows. Section 1 contains some background results from 14. In Section 2, we describe a construction of the model operator $K^{h}$. Section 3 provides some necessary information on magnetic translations and related operator algebras. Finally, in Section 4 we give the proofs of the main results.

I am very thankful to Bernard Helffer for bringing these problems to my attention and useful discussions. 


\section{General Results on equivalence of projections and existence of SPECTRAL GAPS}

In this section we recall general results on equivalence of projections and existence of spectral gaps proved in [14.

Let $\mathfrak{A}$ be a $C^{*}$-algebra, $\mathcal{H}$ a Hilbert space equipped with a faithful $*$-representation of $\mathfrak{A}, \pi: \mathfrak{A} \rightarrow \mathcal{B}(\mathcal{H})$. For simplicity of notation, we will often identify the algebra $\mathfrak{A}$ with its image $\pi(\mathfrak{A})$.

Consider Hilbert spaces $\mathcal{H}_{1}$ and $\mathcal{H}_{2}$ equipped with inner products $(\cdot, \cdot)_{1}$ and $(\cdot, \cdot)_{2}$. Assume that there are given unitary operators $\mathcal{V}_{1}: \mathcal{H}_{1} \rightarrow \mathcal{H}$ and $\mathcal{V}_{2}: \mathcal{H}_{2} \rightarrow \mathcal{H}$. Using the unitary isomorphisms $\mathcal{V}_{1}$ and $\mathcal{V}_{2}$, we get representations $\pi_{1}$ and $\pi_{2}$ of $\mathfrak{A}$ in $\mathcal{H}_{1}$ and $\mathcal{H}_{2}$ accordingly, $\pi_{l}(a)=\mathcal{V}_{l}^{-1} \circ \pi(a) \circ \mathcal{V}_{l}, l=1,2, a \in \mathfrak{A}$.

Consider (unbounded) self-adjoint operators $A_{1}$ in $\mathcal{H}_{1}$ and $A_{2}$ in $\mathcal{H}_{2}$ with the domains $\operatorname{Dom}\left(A_{1}\right)$ and $\operatorname{Dom}\left(A_{2}\right)$ respectively. We will assume that

- the operators $A_{1}$ and $A_{2}$ are semi-bounded from below:

$$
\begin{array}{ll}
\left(A_{1} u, u\right)_{1} \geq \lambda_{01}\|u\|_{1}^{2}, & u \in \operatorname{Dom}\left(A_{1}\right), \\
\left(A_{2} u, u\right)_{2} \geq \lambda_{02}\|u\|_{2}^{2}, & u \in \operatorname{Dom}\left(A_{2}\right),
\end{array}
$$

with some $\lambda_{01}, \lambda_{02} \leq 0$;

- for any $t>0$, the operators $e^{-t A_{l}}, l=1,2$, belong to $\pi_{l}(\mathfrak{A})$.

Let $\mathcal{H}_{0}$ be a Hilbert space, equipped with injective bounded linear maps $i_{1}: \mathcal{H}_{0} \rightarrow \mathcal{H}_{1}$ and $i_{2}: \mathcal{H}_{0} \rightarrow \mathcal{H}_{2}$. Assume that there are given bounded linear maps $p_{1}: \mathcal{H}_{1} \rightarrow \mathcal{H}_{0}$ and $p_{2}: \mathcal{H}_{2} \rightarrow \mathcal{H}_{0}$ such that $p_{1} \circ i_{1}=\mathrm{id}_{\mathcal{H}_{0}}$ and $p_{2} \circ i_{2}=\mathrm{id}_{\mathcal{H}_{0}}$. The whole picture can be represented by the following diagram (note that this diagram is not commutative).

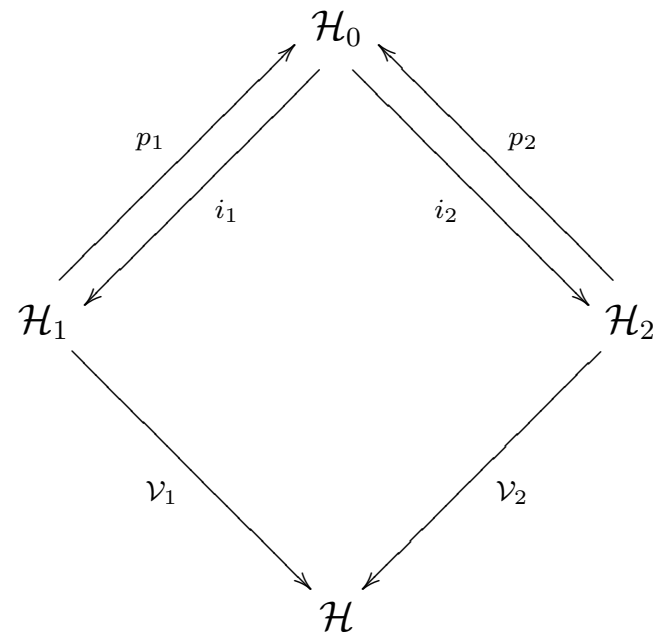

Consider a self-adjoint bounded operator $J$ in $\mathcal{H}_{0}$. We assume that

- the operator $\mathcal{V}_{2} i_{2} J p_{1} \mathcal{V}_{1}^{-1}$ belongs to the von Neumann algebra $\pi(\mathfrak{A})^{\prime \prime}$;

- $\left(i_{2} J p_{1}\right)^{*}=i_{1} J p_{2}$; 
- for any $a \in \mathfrak{A}$, the operator $\pi(a) \mathcal{V}_{2}\left(i_{2} J p_{1}\right) \mathcal{V}_{1}^{-1}$ belongs to $\pi(\mathfrak{A})$.

Since the operators $i_{l}: \mathcal{H}_{0} \rightarrow \mathcal{H}_{l}, l=1,2$, are bounded and have bounded left-inverse operators $p_{l}$, they are topological monomorphisms, i.e. they have closed image and the maps $i_{l}: \mathcal{H}_{0} \rightarrow \operatorname{Im} i_{l}$ are topological isomorphisms. Therefore, we can assume that the estimate

$$
\rho^{-1}\left\|i_{2} J u\right\|_{2} \leq\left\|i_{1} J u\right\|_{1} \leq \rho\left\|i_{2} J u\right\|_{2}, \quad u \in \mathcal{H}_{0},
$$

holds with some $\rho>1$ (depending on $J$ ).

Define the bounded operators $J_{l}$ in $\mathcal{H}_{l}, l=1,2$, by the formula $J_{l}=i_{l} J p_{l}$. We assume that

- the operator $J_{l}, l=1,2$, maps the domain of $A_{l}$ to itself;

- $J_{l}$ is self-adjoint, and $0 \leq J_{l} \leq \mathrm{id}_{\mathcal{H}_{l}}, l=1,2$;

- for $u \in \mathcal{H}_{0}, i_{1} J u \in \operatorname{Dom}\left(A_{1}\right)$ iff $i_{2} J u \in \operatorname{Dom}\left(A_{2}\right)$.

Denote $D=\left\{u \in \mathcal{H}_{0}: i_{1} J u \in \operatorname{Dom}\left(A_{1}\right)\right\}=\left\{u \in \mathcal{H}_{0}: i_{2} J u \in \operatorname{Dom}\left(A_{2}\right)\right\}$.

Introduce a self-adjoint positive bounded linear operator $J_{l}^{\prime}$ in $\mathcal{H}_{l}$ by the formula $J_{l}^{2}+J_{l}^{\prime 2}=\mathrm{id}_{\mathcal{H}_{l}}$. We assume that

- the operator $J_{l}^{\prime}, l=1,2$, maps the domain of $A_{l}$ to itself;

- the operators $\left[J_{l},\left[J_{l}, A_{l}\right]\right]$ and $\left[J_{l}^{\prime},\left[J_{l}^{\prime}, A_{l}\right]\right]$ extend to bounded operators in $\mathcal{H}_{l}$, and

$$
\max \left(\left\|\left[J_{l},\left[J_{l}, A_{l}\right]\right]\right\|_{l},\left\|\left[J_{l}^{\prime},\left[J_{l}^{\prime}, A_{l}\right]\right]\right\|_{l}\right) \leq \gamma_{l}, \quad l=1,2 .
$$

Finally, we assume that

$$
\left(A_{l} J_{l}^{\prime} u, J_{l}^{\prime} u\right)_{l} \geq \alpha_{l}\left\|J_{l}^{\prime} u\right\|_{l}^{2}, \quad u \in \operatorname{Dom}\left(A_{l}\right), \quad l=1,2,
$$

for some $\alpha_{l}>0$, and

$$
\begin{array}{ll}
\left(A_{2} i_{2} J u, i_{2} J u\right)_{2} \leq \beta_{1}\left(A_{1} i_{1} J u, i_{1} J u\right)_{1}+\varepsilon_{1}\left\|i_{1} J u\right\|_{1}^{2}, & u \in D, \\
\left(A_{1} i_{1} J u, i_{1} J u\right)_{1} \leq \beta_{2}\left(A_{2} i_{2} J u, i_{2} J u\right)_{2}+\varepsilon_{2}\left\|i_{2} J u\right\|_{2}^{2}, & u \in D,
\end{array}
$$

for some $\beta_{1}, \beta_{2} \geq 1$ and $\varepsilon_{1}, \varepsilon_{2}>0$.

Denote by $E_{l}(\lambda), l=1,2$, the spectral projection of the operator $A_{l}$, corresponding to the semi-axis $(-\infty, \lambda]$. We assume that there exists a faithful, normal, semi-finite trace $\tau$ on $\pi(\mathfrak{A})^{\prime \prime}$ such that, for any $t>0$, the operators $\mathcal{V}_{l} e^{-t A_{l}} \mathcal{V}_{l}^{-1}, l=1,2$, belong to $\pi(\mathfrak{A})$ and have finite trace. By standard arguments, it follows that $\mathcal{V}_{l} E_{l}(\lambda) \mathcal{V}_{l}^{-1} \in \pi(\mathfrak{A})^{\prime \prime}$, and $\tau\left(\mathcal{V}_{l} E_{l}(\lambda) \mathcal{V}_{l}^{-1}\right)<\infty$ for any $\lambda, l=1,2$.

Theorem 3. Under current assumptions, let $b_{1}>a_{1}$ and

$$
\begin{aligned}
a_{2} & =\rho\left[\beta_{1}\left(a_{1}+\gamma_{1}+\frac{\left(a_{1}+\gamma_{1}-\lambda_{01}\right)^{2}}{\alpha_{1}-a_{1}-\gamma_{1}}\right)+\varepsilon_{1}\right], \\
b_{2} & =\frac{\beta_{2}^{-1}\left(b_{1} \rho^{-1}-\varepsilon_{2}\right)\left(\alpha_{2}-\gamma_{2}\right)-\alpha_{2} \gamma_{2}+2 \lambda_{02} \gamma_{2}-\lambda_{02}^{2}}{\alpha_{2}-2 \lambda_{02}+\beta_{2}^{-1}\left(b_{1} \rho^{-1}-\varepsilon_{2}\right)} .
\end{aligned}
$$

Suppose that $\alpha_{1}>a_{1}+\gamma_{1}, \alpha_{2}>b_{2}+\gamma_{2}$ and $b_{2}>a_{2}$. If the interval $\left(a_{1}, b_{1}\right)$ does not intersect with the spectrum of $A_{1}$, then: 
(1) the interval $\left(a_{2}, b_{2}\right)$ does not intersect with the spectrum of $A_{2}$;

(2) for any $\lambda_{1} \in\left(a_{1}, b_{1}\right)$ and $\lambda_{2} \in\left(a_{2}, b_{2}\right)$, the projections $\mathcal{V}_{1} E_{1}\left(\lambda_{1}\right) \mathcal{V}_{1}^{-1}$ and $\mathcal{V}_{2} E_{2}\left(\lambda_{2}\right) \mathcal{V}_{2}^{-1}$ belong to $\mathfrak{A}$ and are Murray-von Neumann equivalent in $\mathfrak{A}$.

Remark 4. Since $\rho>1, \beta_{1} \geq 1, \gamma_{1}>0$ and $\varepsilon_{1}>0$, we, clearly, have $a_{2}>a_{1}$. The formula (91) is equivalent to the formula

$$
b_{1}=\rho\left[\beta_{2}\left(b_{2}+\gamma_{2}+\frac{\left(b_{2}+\gamma_{2}-\lambda_{02}\right)^{2}}{\alpha_{2}-b_{2}-\gamma_{2}}\right)+\varepsilon_{2}\right],
$$

which is obtained from (8), if we replace $\alpha_{1}, \beta_{1}, \gamma_{1}, \varepsilon_{1}, \lambda_{01}$ by $\alpha_{2}, \beta_{2}, \gamma_{2}, \varepsilon_{2}, \lambda_{02}$ accordingly and $a_{1}$ and $a_{2}$ by $b_{2}$ and $b_{1}$ accordingly. In particular, this implies that $b_{1}>b_{2}$.

\section{THE MODEL OPERATOR}

Here we will give a construction of the model operator, using ideas of [5]. We will use notation of Introduction. Choose a fundamental domain $\mathcal{F} \subset \widetilde{M}$ so that there is no zeros of $B$ on the boundary of $\mathcal{F}$. This is equivalent to saying that the translations $\{\gamma \mathcal{F}, \gamma \in \Gamma\}$ cover the set of all zeros of $B$. Let $\left\{\bar{x}_{j} \mid j=1, \ldots, N\right\}$ denote all the zeros of $B$ in $\mathcal{F} ; \bar{x}_{i} \neq \bar{x}_{j}$ if $i \neq j$.

The model operator $K^{h}$ associated with $H^{h}$ is an operator in $L^{2}\left(\mathbb{R}^{n}\right)^{N}$ given by

$$
K^{h}=\oplus_{1 \leq j \leq N} K_{j}^{h},
$$

where $K_{j}^{h}$ is an unbounded self-adjoint differential operator in $L^{2}\left(\mathbb{R}^{n}\right)$ which corresponds to the zero $\bar{x}_{j}$. Let us fix local coordinates $f_{j}: U\left(\bar{x}_{j}\right) \rightarrow \mathbb{R}^{n}$ on $\widetilde{M}$ defined in a small neighborhood $U\left(\bar{x}_{j}\right)$ of $\bar{x}_{j}$ for every $j=1, \ldots, N$. We assume that $f_{j}\left(\bar{x}_{j}\right)=0$ and the image $f_{j}\left(U\left(\bar{x}_{j}\right)\right)$ is a fixed ball $B=B(0, r) \subset \mathbb{R}^{n}$ centered at the origin 0 .

Write the 2-form $\mathbf{B}$ in the local coordinates as

$$
\mathbf{B}_{j}(X)=\sum_{1 \leq l<m \leq n} b_{l m}(X) d X_{l} \wedge d X_{m}, \quad X=\left(X_{1}, \ldots, X_{n}\right) \in B(0, r) .
$$

The 1-form $\mathbf{A}$ is written in the local coordinates as a 1-form $\mathbf{A}_{j}$ on $B(0, r)$. By 4 , there exists a real function $\theta_{j} \in C^{\infty}(B(0, r))$ such that

$$
\left|\mathbf{A}_{j}(X)-d \theta_{j}(X)\right| \leq C|X|^{k+1}, \quad X \in B(0, r) .
$$

Write the 1-form $\mathbf{A}_{j}-d \theta_{j}$ as

$$
\mathbf{A}_{j}(X)-d \theta_{j}(X)=\sum_{l=1}^{n} a_{l}(X) d X_{l}, \quad X \in B(0, r) .
$$

Let $\mathbf{A}_{1, j}$ be a 1 -form on $\mathbb{R}^{n}$ with polynomial coefficients given by

$$
\mathbf{A}_{1, j}(X)=\sum_{l=1}^{n} \sum_{|\alpha|=k+1} \frac{X^{\alpha}}{\alpha !} \frac{\partial^{\alpha} a_{l}}{\partial X^{\alpha}}(0) d X_{l}, \quad X \in \mathbb{R}^{n}
$$


So we have

$$
d \mathbf{A}_{1, j}(X)=\mathbf{B}_{j}^{0}(X), \quad X \in \mathbb{R}^{n},
$$

where $\mathbf{B}_{j}^{0}$ is a closed 2 -form on $\mathbb{R}^{n}$ with polynomial coefficients defined by

$$
\mathbf{B}_{j}^{0}(X)=\sum_{1 \leq l<m \leq n} \sum_{|\alpha|=k} \frac{X^{\alpha}}{\alpha !} \frac{\partial^{\alpha} b_{l m}}{\partial X^{\alpha}}(0) d X_{l} \wedge d X_{m}, \quad X \in \mathbb{R}^{n} .
$$

Take any extension of the function $\theta_{j}$ to a smooth, compactly supported function in $\mathbb{R}^{n}$ denoted also by $\theta_{j}$ and put

$$
\mathbf{A}_{j}^{0}(X)=\mathbf{A}_{1, j}(X)+d \theta_{j}(X), \quad X \in \mathbb{R}^{n} .
$$

Then we still have

$$
d \mathbf{A}_{j}^{0}(X)=\mathbf{B}_{j}^{0}(X), \quad X \in \mathbb{R}^{n},
$$

and, moreover,

$$
\begin{aligned}
& \left|\mathbf{B}_{j}(X)-\mathbf{B}_{j}^{0}(X)\right| \leq C|X|^{k+1}, \quad X \in B(0, r), \\
& \left|\mathbf{A}_{j}(X)-\mathbf{A}_{j}^{0}(X)\right| \leq C|X|^{k+2}, \quad X \in B(0, r) .
\end{aligned}
$$

Then $K_{j}^{h}$ is the self-adjoint differential operator with asymptotically polynomial coefficients in $L^{2}\left(\mathbb{R}^{n}\right)$ given by

$$
K_{j}^{h}=\left(i h d+\mathbf{A}_{j}^{0}\right)^{*}\left(i h d+\mathbf{A}_{j}^{0}\right),
$$

where the adjoint is taken with respect to a Hilbert structure in $L^{2}\left(\mathbb{R}^{n}\right)$ given by the flat Riemannian metric $\left(g_{l m}(0)\right)$ in $\mathbb{R}^{n}$. If we write $\mathbf{A}_{j}^{0}$ as

$$
\mathbf{A}_{j}^{0}=A_{j, 1}^{0} d X_{1}+\ldots+A_{j, n}^{0} d X_{n},
$$

then $K_{j}^{h}$ is given as

$$
K_{j}^{h}=\sum_{1 \leq l, m \leq n} g^{l m}(0)\left(i h \frac{\partial}{\partial X_{l}}+A_{j, l}^{0}(X)\right)\left(i h \frac{\partial}{\partial X_{m}}+A_{j, m}^{0}(X)\right) .
$$

The operator $K_{j}^{h}$ has discrete spectrum (cf., for instance, [9, 4]). By gauge invariance, the operator $K_{j}^{h}$ is unitarily equivalent to the Schrödinger operator

$$
H_{j}^{h}=\left(i h d+\mathbf{A}_{1, j}\right)^{*}\left(i h d+\mathbf{A}_{1, j}\right),
$$

associated with the homogeneous 1-form $\mathbf{A}_{1, j}$. Using a simple scaling $X \mapsto h^{\frac{1}{k+2}} X$, it can be shown that the operator $H_{j}^{h}$ is unitarily equivalent to the operator $h^{\frac{2 k+2}{k+2}} H_{j}^{1}$. So we conclude that the operator $h^{-\frac{2 k+2}{k+2}} K^{h}$ has discrete spectrum independent of $h$, which is denoted by $\left\{\lambda_{m}: m \in \mathbb{N}\right\}, \lambda_{1}<\lambda_{2}<\lambda_{2}<\ldots$ (not taking into account multiplicities). As it will be shown in Section 4 , the sequence $\left\{\lambda_{m}: m \in \mathbb{N}\right\}$ is precisely what we need for the proof of Theorem 1 


\section{Magnetic translations And RElated operator Algebras}

In this section, we collect some necessary facts on magnetic translations and related operator algebras (see, for instance, [15, 14] and references therein for more details). As above, let $M$ be a compact connected Riemannian manifold, $\Gamma$ be its fundamental group and $p: \widetilde{M} \rightarrow M$ be its universal cover. Let $\mathbf{B}$ be a closed $\Gamma$-invariant real-valued 2 -form on $M$. Assume that $\mathbf{B}$ is exact. So $\mathbf{B}=d \mathbf{A}$ where $\mathbf{A}$ is a 1 -form on $\widetilde{M}$. We will assume without loss of generality that $\mathbf{A}$ is real-valued.

The Hermitian connection $\mathbf{A}$ defines a projective $(\Gamma, \sigma)$-unitary representation on $L^{2}(\widetilde{M})$, that is, the map $T: \Gamma \rightarrow \mathcal{U}\left(L^{2}(\widetilde{M})\right), \gamma \mapsto T_{\gamma}$, where for any Hilbert space $\mathcal{H}$ we denote by $\mathcal{U}(\mathcal{H})$ the group of all unitary operators in $\mathcal{H}$, satisfying

$$
T_{e}=\mathrm{id}, \quad T_{\gamma_{1}} T_{\gamma_{2}}=\sigma\left(\gamma_{1}, \gamma_{2}\right) T_{\gamma_{1} \gamma_{2}}, \quad \gamma_{1}, \gamma_{2} \in \Gamma .
$$

Here $\sigma$ is a multiplier on $\Gamma$ i.e. $\sigma: \Gamma \times \Gamma \rightarrow U(1)$ satisfies

- $\sigma(\gamma, e)=\sigma(e, \gamma)=1, \quad \gamma \in \Gamma$;

- $\sigma\left(\gamma_{1}, \gamma_{2}\right) \sigma\left(\gamma_{1} \gamma_{2}, \gamma_{3}\right)=\sigma\left(\gamma_{1}, \gamma_{2} \gamma_{3}\right) \sigma\left(\gamma_{2}, \gamma_{3}\right), \quad \gamma_{1}, \gamma_{2}, \gamma_{3} \in \Gamma \quad$ (the cocycle relation).

In other words one says that the map $\gamma \mapsto T_{\gamma}$ defines a $(\Gamma, \sigma)$-action in $\mathcal{H}$. The operators $T_{\gamma}$ are also called magnetic translations.

Denote by $\ell^{2}(\Gamma)$ the standard Hilbert space of complex-valued $L^{2}$-functions on the discrete group $\Gamma$. For any $\gamma \in \Gamma$, define a bounded operator $T_{\gamma}^{L}$ in $\ell^{2}(\Gamma)$ by

$$
T_{\gamma}^{L} f\left(\gamma^{\prime}\right)=f\left(\gamma^{-1} \gamma^{\prime}\right) \bar{\sigma}\left(\gamma, \gamma^{-1} \gamma^{\prime}\right), \quad \gamma^{\prime} \in \Gamma, \quad f \in \ell^{2}(\Gamma)
$$

It is easy to see that

$$
T_{e}^{L}=\mathrm{id}, \quad T_{\gamma_{1}}^{L} T_{\gamma_{2}}^{L}=\bar{\sigma}\left(\gamma_{1}, \gamma_{2}\right) T_{\gamma_{1} \gamma_{2}}^{L}, \quad \gamma_{1}, \gamma_{2} \in \Gamma
$$

Also

$$
\left(T_{\gamma}^{L}\right)^{*}=\sigma\left(\gamma, \gamma^{-1}\right) T_{\gamma^{-1}}^{L}
$$

This means that $T_{\gamma}$ is a left $(\Gamma, \bar{\sigma})$-action on $\ell^{2}(\Gamma)$ (or, equivalently, a $(\Gamma, \bar{\sigma})$-unitary representation in $\left.\ell^{2}(\Gamma)\right)$.

Define a twisted group algebra $\mathbb{C}(\Gamma, \bar{\sigma})$ which consists of complex valued functions with finite support on $\Gamma$, with the twisted convolution operation

$$
(f * g)(\gamma)=\sum_{\gamma_{1}, \gamma_{2}: \gamma_{1} \gamma_{2}=\gamma} f\left(\gamma_{1}\right) g\left(\gamma_{2}\right) \bar{\sigma}\left(\gamma_{1}, \gamma_{2}\right)
$$

and with the involution

$$
f^{*}(\gamma)=\sigma\left(\gamma, \gamma^{-1}\right) \overline{f\left(\gamma^{-1}\right)}
$$

Associativity of the multiplication is equivalent to the cocycle condition. The basis of $\mathbb{C}(\Gamma, \bar{\sigma})$ as a vector space is formed by $\delta$-functions $\left\{\delta_{\gamma}\right\}_{\gamma \in \Gamma}, \delta_{\gamma}\left(\gamma^{\prime}\right)=1$ if $\gamma=\gamma^{\prime}$ and 0 otherwise. We have

$$
\delta_{\gamma_{1}} * \delta_{\gamma_{2}}=\bar{\sigma}\left(\gamma_{1}, \gamma_{2}\right) \delta_{\gamma_{1} \gamma_{2}}
$$


Note also that the $\delta$-functions $\left\{\delta_{\gamma}\right\}_{\gamma \in \Gamma}$ form an orthonormal basis in $\ell^{2}(\Gamma)$. It is easy to check that

$$
T_{\gamma}^{L} \delta_{\gamma^{\prime}}=\delta_{\gamma} * \delta_{\gamma^{\prime}}=\bar{\sigma}\left(\gamma, \gamma^{\prime}\right) \delta_{\gamma \gamma^{\prime}}
$$

It is clear that the correspondence $f \in \mathbb{C}(\Gamma, \bar{\sigma}) \mapsto T^{L}(f) \in \mathcal{B}\left(\ell^{2}(\Gamma)\right)$, where $T^{L}(f) u=$ $f * u, u \in \ell^{2}(\Gamma)$, defines a $*$-representation of the twisted group algebra $\mathbb{C}(\Gamma, \bar{\sigma})$ in $\ell^{2}(\Gamma)$. The weak closure of the image of $\mathbb{C}(\Gamma, \bar{\sigma})$ in this representation coincides with the (left) twisted group von Neumann algebra $\mathcal{A}^{L}(\Gamma, \bar{\sigma})$. The corresponding norm closure is so called reduced twisted group $C^{*}$-algebra which is denoted $C_{r}^{*}(\Gamma, \bar{\sigma})$.

The von Neumann algebra $\mathcal{A}^{L}(\Gamma, \bar{\sigma})$ can be described in terms of the matrix elements. For any $A \in \mathcal{B}\left(\ell^{2}(\Gamma)\right)$ denote $A_{x, y}=\left(A \delta_{y}, \delta_{x}\right), x, y \in \Gamma$ (which is a matrix element of $A$ ). Then repeating standard arguments (given in a similar situation e.g. in [16) we can prove that for any $A \in \mathcal{B}\left(\ell^{2}(\Gamma)\right)$ the inclusion $A \in \mathcal{A}^{L}(\Gamma, \bar{\sigma})$ is equivalent to the relations

$$
A_{x \gamma, y \gamma}=\bar{\sigma}(x, \gamma) \sigma(y, \gamma) A_{x, y}, \quad x, y, \gamma \in \Gamma .
$$

In particular, for any $A \in \mathcal{A}^{L}(\Gamma, \bar{\sigma})$, we have

$$
A_{x \gamma, x \gamma}=A_{x, x}, \quad x, \gamma \in \Gamma \text {. }
$$

A finite von Neumann trace $\operatorname{tr}_{\Gamma, \bar{\sigma}}: \mathcal{A}^{L}(\Gamma, \bar{\sigma}) \rightarrow \mathbb{C}$ is defined by the formula

$$
\operatorname{tr}_{\Gamma, \bar{\sigma}} A=\left(A \delta_{e}, \delta_{e}\right) \text {. }
$$

We can also write $\operatorname{tr}_{\Gamma, \bar{\sigma}} A=A_{\gamma, \gamma}=\left(A \delta_{\gamma}, \delta_{\gamma}\right)$ for any $\gamma \in \Gamma$ because the right hand side does not depend of $\gamma$.

\section{Proof of MAin ReSUlts}

For the proof of the main theorem, we apply Theorem 3 in a following particular setting. Take the $C^{*}$ algebra $\mathfrak{A}$ to be $C_{r}^{*}(\Gamma, \bar{\sigma}) \otimes \mathcal{K}$. Let $\mathcal{H}$ be the Hilbert space $\ell^{2}(\Gamma) \otimes$ $\ell^{2}(\mathbb{N})$. Put $\mathcal{H}_{1}=\ell^{2}(\Gamma) \otimes L^{2}\left(\mathbb{R}^{n}\right)^{N}$ and $\mathcal{H}_{2}=L^{2}(\widetilde{M})$. Choose an arbitrary unitary isomorphism $V_{1}: L^{2}\left(\mathbb{R}^{n}\right)^{N} \rightarrow \ell^{2}(\mathbb{N})$ and define an unitary operator $\mathcal{V}_{1}: \mathcal{H}_{1} \rightarrow \mathcal{H}$ as $\mathcal{V}_{1}=\mathrm{id} \otimes V_{1}$.

As in [14, define a $(\Gamma, \sigma)$-equivariant isometry $\mathbf{U}: L^{2}(\widetilde{M}) \cong \ell^{2}(\Gamma) \otimes L^{2}(\mathcal{F})$ by the formula

$$
\mathbf{U}(\phi)=\sum_{\gamma \in \Gamma} \delta_{\gamma} \otimes i^{*}\left(T_{\gamma} \phi\right), \quad \phi \in L^{2}(\widetilde{M}),
$$

where $i: \mathcal{F} \rightarrow \widetilde{M}$ denotes the inclusion map. Choose an arbitrary unitary isomorphism $V_{2}: L^{2}(\mathcal{F}) \rightarrow \ell^{2}(\mathbb{N})$. Then a unitary operator $\mathcal{V}_{2}: \mathcal{H}_{2} \rightarrow \mathcal{H}$ is defined as $\mathcal{V}_{2}=$ $\left(\mathrm{id} \otimes V_{2}\right) \circ \mathbf{U}$.

Let $\pi$ be the representation of the algebra $\mathfrak{A}$ in $\mathcal{H}$ given by the tensor product of the representation $T^{L}$ of $C_{r}^{*}(\Gamma, \bar{\sigma})$ on $\ell^{2}(\Gamma)$ and the standard representation of $\mathcal{K}$ in $\ell^{2}(\mathbb{N})$. So we have $\pi\left(C_{r}^{*}(\Gamma, \bar{\sigma}) \otimes \mathcal{K}\right) \subset \mathcal{A}^{L}(\Gamma, \bar{\sigma}) \otimes \mathcal{B}\left(\ell^{2}(\mathbb{N})\right)$ and $\pi\left(C_{r}^{*}(\Gamma, \bar{\sigma}) \otimes \mathcal{K}\right)^{\prime \prime} \cong$ $\mathcal{A}^{L}(\Gamma, \bar{\sigma}) \otimes \mathcal{B}\left(\ell^{2}(\mathbb{N})\right)$. Using the unitary isomorphisms $\mathcal{V}_{1}$ and $\mathcal{V}_{2}$, we get representations $\pi_{1}$ and $\pi_{2}$ of $\mathfrak{A}$ in $\mathcal{H}_{1}$ and $\mathcal{H}_{2}$ accordingly, $\pi_{l}(a)=\mathcal{V}_{l}^{-1} \circ \pi(a) \circ \mathcal{V}_{l}, l=1,2, a \in \mathfrak{A}$. 
Define a trace $\tau$ on $\mathcal{A}^{L}(\Gamma, \bar{\sigma}) \otimes \mathcal{B}\left(\ell^{2}(\mathbb{N})\right)$ as the tensor product of the finite von Neumann trace $\operatorname{tr}_{\Gamma, \bar{\sigma}}$ on $\mathcal{A}^{L}(\Gamma, \bar{\sigma})$ and the standard trace on $\mathcal{B}\left(\ell^{2}(\mathbb{N})\right)$.

Consider self-adjoint, semi-bounded from below operators $A_{1}$ in $\mathcal{H}_{1}$ and $A_{2}$ in $\mathcal{H}_{2}$ :

$$
A_{1}=\mathrm{id} \otimes h^{-\frac{2 k+2}{k+2}} K^{h}, \quad A_{2}=h^{-\frac{2 k+2}{k+2}} H^{h} .
$$

Clearly, we have

$$
e^{-t A_{1}}=\mathrm{id} \otimes e^{-t h^{-\frac{2 k+2}{k+2}} K^{h}} \in \pi_{1}(\mathfrak{A}) \cong C_{r}^{*}(\Gamma, \bar{\sigma}) \otimes \mathcal{K}\left(\mathfrak{H}_{K}\right)
$$

with $\tau\left(e^{-t A_{1}}\right)<\infty$ for any $t>0$. As shown in [14, for any $t>0$, the operator $e^{-t A_{2}}$ belongs to $\pi_{2}(\mathfrak{A})$ and $\tau\left(e^{-t A_{2}}\right)<\infty$. Remark that, in notation of Theorem 2 ,

$$
E_{1}(\lambda)=\mathrm{id} \otimes E^{0}\left(h^{\frac{2 k+2}{k+2}} \lambda\right), \quad E_{2}(\lambda)=E\left(h^{\frac{2 k+2}{k+2}} \lambda\right) .
$$

We will use notation of Section 2, Let

$$
\mathcal{H}_{0}=\ell^{2}(\Gamma) \otimes\left(\oplus_{j=1}^{N} L^{2}\left(U\left(\bar{x}_{j}\right)\right)\right) .
$$

An inclusion $i_{1}: \mathcal{H}_{0} \rightarrow \mathcal{H}_{1}$ is defined as $i_{1}=\mathrm{id} \otimes j_{1}$, where $j_{1}$ is the inclusion

$$
\oplus_{j=1}^{N} L^{2}\left(U\left(\bar{x}_{j}\right)\right) \cong L^{2}(B(0, r))^{N} \hookrightarrow L^{2}\left(\mathbb{R}^{n}\right)^{N}
$$

given by the chosen local coordinates. An inclusion $i_{2}: \mathcal{H}_{0} \rightarrow \mathcal{H}_{2}$ is defined as $i_{2}=$ $\mathbf{U}^{*} \circ\left(\mathrm{id} \otimes j_{2}\right)$, where $j_{2}$ is the natural inclusion

$$
\oplus_{j=1}^{N} L^{2}\left(U\left(\bar{x}_{j}\right)\right) \hookrightarrow L^{2}(\mathcal{F}) .
$$

The operator $p_{1}: \mathcal{H}_{1} \rightarrow \mathcal{H}_{0}$ is defined as $p_{1}=\mathrm{id} \otimes r_{1}$, where $r_{1}$ is the restriction operator

$$
L^{2}\left(\mathbb{R}^{n}\right)^{N} \rightarrow L^{2}(B(0, r))^{N} \cong \oplus_{j=1}^{N} L^{2}\left(U\left(\bar{x}_{j}\right)\right) .
$$

The operator $p_{2}: \mathcal{H}_{1} \rightarrow \mathcal{H}_{0}$ is defined as $p_{2}=\left(\right.$ id $\left.\otimes r_{2}\right) \circ \mathbf{U}$, where $r_{2}: L^{2}(\mathcal{F}) \rightarrow$ $\oplus_{j=1}^{N} L^{2}\left(U\left(\bar{x}_{j}\right)\right)$ is the restriction operator.

Fix a function $\phi \in C_{c}^{\infty}\left(\mathbb{R}^{n}\right)$ such that $0 \leq \phi \leq 1, \phi(x)=1$ if $|x| \leq 1, \phi(x)=0$ if $|x| \geq 2$, and $\phi^{\prime}=\left(1-\phi^{2}\right)^{1 / 2} \in C^{\infty}\left(\mathbb{R}^{n}\right)$. Fix a number $\kappa>0$, which we shall choose later. For any $h>0$ define $\phi^{(h)}(x)=\phi\left(h^{-\kappa} x\right)$. For any $h>0$ small enough, let $\phi_{j}=\phi^{(h)} \in C_{c}^{\infty}\left(U\left(\bar{x}_{j}\right)\right)$ in the fixed coordinates near $\bar{x}_{j}$. Denote also $\phi_{j, \gamma}=\left(\gamma^{-1}\right)^{*} \phi_{j}$. (This function is supported in the neighborhood $U\left(\gamma \bar{x}_{j}\right)=\gamma\left(U\left(\bar{x}_{j}\right)\right)$ of $\gamma \bar{x}_{j}$.) We will always take $h \in\left(0, h_{0}\right)$ where $h_{0}$ is sufficiently small, so in particular the supports of all functions $\phi_{j, \gamma}$ are disjoint.

Let $\Phi \in C^{\infty}\left(\bigcup_{j=1}^{N} U\left(\bar{x}_{j}\right)\right)$ be equal to $\phi_{j}$ on $U\left(\bar{x}_{j}\right), j=1,2, \ldots, N$. Consider a $(\Gamma, \sigma)$ equivariant, self-adjoint, bounded operator $J$ in $\mathcal{H}_{0}$ defined as $J=$ id $\otimes \Phi$, where $\Phi$ denotes the multiplication operator by the function $\Phi$ in the space $\oplus_{j=1}^{N} L^{2}\left(U\left(\bar{x}_{j}\right)\right)$.

It is clear that

$$
\mathcal{V}_{2} i_{2} J p_{1} \mathcal{V}_{1}^{-1}=\mathrm{id} \otimes V_{2} j_{2} \Phi r_{1} V_{1}^{-1}
$$

and $j_{2} \Phi r_{1}$ is a bounded operator from $L^{2}\left(\mathbb{R}^{n}\right)^{N}$ to $L^{2}(\mathcal{F})$ given as the composition

$$
L^{2}\left(\mathbb{R}^{n}\right)^{N} \rightarrow L^{2}(B(0, r))^{N} \cong \oplus_{j=1}^{N} L^{2}\left(U\left(\bar{x}_{j}\right)\right) \stackrel{\Phi}{\longrightarrow} \oplus_{j=1}^{N} L^{2}\left(U\left(\bar{x}_{j}\right)\right) \hookrightarrow L^{2}(\mathcal{F})
$$


Hence, the operator $\mathcal{V}_{2} i_{2} J p_{1} \mathcal{V}_{1}^{-1}$ belongs to the von Neumann algebra $\pi(\mathfrak{A})^{\prime \prime} \cong \mathcal{A}^{L}(\Gamma, \bar{\sigma}) \otimes$ $\mathcal{B}\left(\ell^{2}(\mathbb{N})\right)$, and, for any $a \in \mathfrak{A}$, the operator $\pi(a) \mathcal{V}_{2}\left(i_{2} J p_{1}\right) \mathcal{V}_{1}^{-1}$ belongs to $\pi(\mathfrak{A})$.

Similarly, we have

$$
i_{1} J p_{2}=\mathrm{id} \otimes j_{1} \Phi r_{2},
$$

and $j_{1} \Phi r_{2}$ is a bounded operator from $L^{2}(\mathcal{F})$ to $L^{2}\left(\mathbb{R}^{n}\right)^{N}$ given as the composition

$$
L^{2}(\mathcal{F}) \rightarrow \oplus_{j=1}^{N} L^{2}\left(U\left(\bar{x}_{j}\right)\right) \stackrel{\Phi}{\longrightarrow} \oplus_{j=1}^{N} L^{2}\left(U\left(\bar{x}_{j}\right)\right) \cong L^{2}(B(0, r))^{N} \hookrightarrow L^{2}\left(\mathbb{R}^{n}\right)^{N} .
$$

So we have $\left(i_{2} J p_{1}\right)^{*}=i_{1} J p_{2}$.

We will use local coordinates near $\bar{x}_{j}$ such that the Riemannian volume element at the point $\bar{x}_{j}$ coincides with the Euclidean volume element given by the chosen local coordinates. Then the estimate (3) holds with

$$
\rho=1+O\left(h^{\kappa}\right) .
$$

Denote by the same letters $\phi$ and $\phi^{\prime}$ the multiplication operators in $L^{2}\left(\mathbb{R}^{n}\right)$ by the functions $\phi$ and $\phi^{\prime}$ accordingly. Let $\Phi_{1}$ and $\Phi_{1}^{\prime}$ be the bounded operators in $L^{2}\left(\mathbb{R}^{n}\right)^{N} \cong$ $L^{2}\left(\mathbb{R}^{n}\right) \otimes \mathbb{C}^{N}$ given by $\Phi_{1}=\phi \otimes \mathrm{id}_{\mathbb{C}^{N}}$ and $\Phi_{1}^{\prime}=\phi^{\prime} \otimes \mathrm{id}_{\mathbb{C}^{N}}$. Then we have $J_{1}=\mathrm{id} \otimes \Phi_{1}$ and $J_{1}^{\prime}=\mathrm{id} \otimes \Phi_{1}^{\prime}$ in $\ell^{2}(\Gamma) \otimes L^{2}\left(\mathbb{R}^{n}\right)^{N}$.

Let $\Phi_{\gamma} \in C^{\infty}(\widetilde{M})$ be equal to $\phi_{j, \gamma}$ on $U\left(\gamma \bar{x}_{j}\right), j=1,2, \ldots, N$, and 0 otherwise. Put $\Phi_{2}=\sum_{\gamma \in \Gamma} \Phi_{\gamma} \in C^{\infty}(\widetilde{M})$. Define a function $\Phi_{2}^{\prime} \in C^{\infty}(\widetilde{M}), \Phi_{2}^{\prime} \geq 0$ by the equation $\left(\Phi_{2}\right)^{2}+\left(\Phi_{2}^{\prime}\right)^{2}=1$ in $C^{\infty}(\widetilde{M})$. The operators $J_{2}$ and $J_{2}^{\prime}$ are given by the multiplication operators by the functions $\Phi_{2}$ and $\Phi_{2}^{\prime}$ in $L^{2}(\widetilde{M})$ respectively.

The estimate (4) hold with

$$
\gamma_{l}=O\left(h^{-\frac{2 k+2}{k+2}+2-2 \kappa}\right), \quad l=1,2 .
$$

Indeed, for any $j=1,2, \ldots, N$, the principal symbol $a_{1, j}^{(2)} \in C^{\infty}\left(T^{*} \mathbb{R}^{n}\right)$ of $K_{j}^{h}$ is given by

$$
a_{1, j}^{(2)}(x, \xi)=h^{2} \sum_{i, k=1}^{n} g^{i k}\left(\bar{x}_{j}\right) \xi_{i} \xi_{k}, \quad(x, \xi) \in T^{*} \mathbb{R}^{n} .
$$

So we have

$$
\left[J_{1},\left[J_{1}, A_{1}\right]\right]=-h^{-\frac{2 k+2}{k+2}+2} \mathrm{id} \otimes\left(\oplus_{1 \leq j \leq N} a_{1, j}^{(2)}(x, d \phi(x))\right)
$$

and

$$
\left[J_{1}^{\prime},\left[J_{1}^{\prime}, A_{1}\right]\right]=-h^{-\frac{2 k+2}{k+2}+2} \mathrm{id} \otimes\left(\oplus_{1 \leq j \leq N} a_{1, j}^{(2)}\left(x, d \phi^{\prime}(x)\right)\right)
$$

in $\ell^{2}(\Gamma) \otimes L^{2}\left(\mathbb{R}^{n}\right)^{N}$. Similarly, the principal symbol $a_{2}^{(2)} \in C^{\infty}\left(T^{*} \widetilde{M}\right)$ of $H^{h}$ is given by

$$
a_{2}^{(2)}(x, \xi)=h^{2} \sum_{i, k=1}^{n} g^{i k}(x) \xi_{i} \xi_{k}, \quad(x, \xi) \in T^{*} \widetilde{M}
$$


So the operators $\left[J_{2},\left[J_{2}, A_{2}\right]\right],\left[J_{2}^{\prime},\left[J_{2}^{\prime}, A_{2}\right]\right]$ are the multiplication operators in $L^{2}(\widetilde{M})$ by the functions $-h^{-\frac{2 k+2}{k+2}+2} a_{2}^{(2)}\left(x, d \Phi_{2}(x)\right)$ and $-h^{-\frac{2 k+2}{k+2}+2} a_{2}^{(2)}\left(x, d \Phi_{2}^{\prime}(x)\right)$ accordingly. Therefore,

$$
\begin{aligned}
\gamma_{1} & =h^{-\frac{2 k+2}{k+2}+2} \max _{j=1,2, \ldots, N} \max \left(\sup _{x \in \mathbb{R}^{n}}\left(a_{1, j}^{(2)}(x, d \phi(x))\right), \sup _{x \in \mathbb{R}^{n}}\left(a_{1, j}^{(2)}\left(x, d \phi^{\prime}(x)\right)\right)\right) \\
& =O\left(h^{-\frac{2 k+2}{k+2}+2-2 \kappa}\right), \\
\gamma_{2} & =h^{-\frac{2 k+2}{k+2}+2} \max \left(\sup _{x \in \widetilde{M}}\left(a_{2}^{(2)}\left(x, d \Phi_{2}(x)\right)\right), \sup _{x \in \widetilde{M}}\left(a_{2}^{(2)}\left(x, d \Phi_{2}^{\prime}(x)\right)\right)\right) \\
& =O\left(h^{-\frac{2 k+2}{k+2}+2-2 \kappa}\right) .
\end{aligned}
$$

The estimates (5) hold with

$$
\alpha_{l}=O\left(h^{-\frac{2 k+2}{k+2}+k \kappa+1}\right), \quad l=1,2 .
$$

Indeed, let $q_{0, j}^{h}$ denote the quadratic hermitian form associated to $K_{j}^{h}$,

$$
q_{0, j}^{h}(u)=\left(K_{j}^{h} u, u\right)=\int_{\mathbb{R}^{n}}\left|i h d u+\mathbf{A}_{j}^{0} u\right|^{2} \sqrt{g(0)} d x .
$$

Consider the operator $H_{j}^{h}=\left(i h d+\mathbf{A}_{1, j}\right)^{*}\left(i h d+\mathbf{A}_{1, j}\right)$ and denote by $q_{1, j}^{h}$ the quadratic hermitian form associated to this operator:

$$
q_{1, j}^{h}(u)=\left(H_{j}^{h} u, u\right)=\int_{\mathbb{R}^{n}}\left|i h d u+\mathbf{A}_{1, j} u\right|^{2} \sqrt{g(0)} d x,
$$

By [5, Theorem 4.4], there exists a constant $C_{j}>0$ such that for any $h>0$,

$$
h \int_{\mathbb{R}^{n}}\left|B_{0, j}(x)\right||u(x)|^{2} d x \leq C_{j} q_{1, j}^{h}(u), \quad u \in C_{c}^{\infty}\left(\mathbb{R}^{n}\right) .
$$

Using gauge invariance and (15), we get

$$
q_{0, j}^{h}(u)=q_{1, j}^{h}\left(e^{i \theta_{j}} u\right) \geq \frac{h}{C_{j}} \int_{\mathbb{R}^{n}}\left|B_{0, j}(x)\right|\left|e^{i \theta_{j}} u(x)\right|^{2} d x=\frac{h}{C_{j}} \int_{\mathbb{R}^{n}}\left|B_{0, j}(x)\right||u(x)|^{2} d x .
$$

Similarly, let $q^{h}$ be the quadratic hermitian form associated to $H^{h}$,

$$
q^{h}(u)=\left(H^{h} u, u\right)=\int_{\widetilde{M}}|i h d u+\mathbf{A} u|^{2} d \mu(x),
$$

where $d \mu$ denotes the Riemannian volume form on $\widetilde{M}$. By an easy modification of the proof of Theorem 4.5 in [5], one can show that there exists a constant $C_{0}>0$, and for any $\varepsilon \in(0,1)$, there exists a constant $C_{\varepsilon}>0$ such that for any $h \in\left(0, h_{0}\right]$,

$$
h \int_{\widetilde{M}}|B(x)||u(x)|^{2} d \mu(x) \leq C_{0}\left[q^{h}(u)+C_{\varepsilon} h^{2-\varepsilon}\|u\|^{2}\right], \quad u \in C_{c}^{\infty}(\widetilde{M}) .
$$

Assume that $k \kappa<2$ and take $\varepsilon \in(0,1)$ so that $k \kappa<2-\varepsilon$. Since $|B(x)| \geq C h^{k \kappa}$ for $x \in \operatorname{supp} \Phi_{l}^{\prime}, l=1,2$, the estimates (16) and (17) easily imply the desired estimates (5). 
The constants $\lambda_{0 l}, l=1,2$, can be chosen to be independent of $h$. Namely, one can take

$$
\lambda_{01}=\lambda_{02}=0 .
$$

Finally, the estimates (6) and (17) hold with

$$
\beta_{l}=1+O\left(h^{\kappa}\right), \quad \varepsilon_{l}=O\left(h^{2 \kappa(k+2)-\kappa-\frac{2 k+2}{k+2}}\right), \quad l=1,2 .
$$

Using the inequality $|a+b|^{2} \leq|a|^{2}+2|a||b|+|b|^{2} \leq(1+\varepsilon)|a|^{2}+\left(1+\varepsilon^{-1}\right)|b|^{2}$ with $\varepsilon=h^{\kappa}$, we get

$$
\begin{aligned}
q^{h}(\phi u)= & \left(H^{h}(\phi u), \phi u\right)=\int_{U\left(\bar{x}_{j}\right)}|i h d(\phi u)+\mathbf{A} \phi u|^{2} d \mu(x) \\
\leq & \left(1+h^{\kappa}\right) \int_{B(0, r)}\left|i h d(\phi u)+\mathbf{A}_{j} \phi u\right|^{2} \sqrt{g(0)} d x \\
\leq & \left(1+h^{\kappa}\right) \int_{B(0, r)}\left|i h d(\phi u)+\mathbf{A}_{j}^{0} \phi u\right|^{2} \sqrt{g(0)} d x \\
& +c h^{-\kappa} \int_{B(0, r)}\left|\left(\mathbf{A}_{j}-\mathbf{A}_{j}^{0}\right) \phi u\right|^{2} \sqrt{g(0)} d x .
\end{aligned}
$$

By (11), we have

$$
\int_{B(0, r)}\left|\left(\mathbf{A}_{j}-\mathbf{A}_{j}^{0}\right) \phi u\right|^{2} \sqrt{g(0)} d x \leq C h^{2 \kappa(k+2)} \int_{B(0, r)}|\phi u|^{2} \sqrt{g(0)} d x,
$$

that completes the proof of (6). The proof of (7) is similar.

Now we complete the proofs of Theorems 1 and 2. As above, take $\left\{\lambda_{m}: m \in \mathbb{N}\right\}$, $\lambda_{1}<\lambda_{2}<\lambda_{2}<\ldots$, to be the spectrum of the operator $h^{-\frac{2 k+2}{k+2}} K^{h}$ (without taking into account multiplicities), which is independent of $h$. Take any $a$ and $b$ such that $\lambda_{m}<a<b<\lambda_{m+1}$ with some $m$. Clearly, the spectrum of the operator $A_{1}$ coincides with the spectrum of the operator $h^{-\frac{2 k+2}{k+2}} K^{h}$. Therefore, the interval $[a, b]$ does not intersect with the spectrum of $A_{1}$. Take any open interval $\left(a_{1}, b_{1}\right)$ that contains $[a, b]$ and does not intersect with the spectrum of $A_{1}$. Using the estimates (12), (13), (14), (18) and (19), one can see that, for $a_{2}$ and $b_{2}$ given by (8) and (9), we have

$$
a_{2}=a_{1}+O\left(h^{s}\right), \quad b_{2}=b_{1}+O\left(h^{s}\right), \quad h \rightarrow 0,
$$

where $s=\min \left\{(2 k+3) \kappa-\frac{2 k+2}{k+2},-\frac{2 k+2}{k+2}+2-2 \kappa\right\}$. The best possible value of $s$ which is

$$
s=\max _{\kappa} \min \left\{(2 k+3) \kappa-\frac{2 k+2}{k+2},-\frac{2 k+2}{k+2}+2-2 \kappa\right\}=\frac{2}{(2 k+5)(k+2)}
$$

is attained when $\kappa=\frac{2}{2 k+5}$.

Hence, if $h>0$ is small enough, we have $\alpha_{1}>a_{1}+\gamma_{1}, \alpha_{2}>b_{2}+\gamma_{2}, b_{2}>a_{2}$ and the interval $\left(a_{2}, b_{2}\right)$ contains $[a, b]$. By Theorem 3] we conclude that the interval $\left(a_{2}, b_{2}\right)$ does not intersect with the spectrum of $A_{2}$, that completes the proof of Theorem 1. Moreover, we have that, for any $\lambda_{1} \in\left(a_{1}, b_{1}\right)$ and $\lambda_{2} \in\left(a_{2}, b_{2}\right)$, the spectral projections $\mathcal{V}_{1} E_{1}\left(\lambda_{1}\right) \mathcal{V}_{1}^{-1}$ 
and $\mathcal{V}_{2} E_{2}\left(\lambda_{2}\right) \mathcal{V}_{2}^{-1}$ are equivalent in $\mathfrak{A}$. Putting $U=\mathcal{V}_{1}^{-1} \mathcal{V}_{2}$, we get the desired Murray von Neumann equivalence of $E_{1}\left(\lambda_{1}\right)=\mathrm{id} \otimes E^{0}(\lambda)$ and $\mathcal{V}_{1}^{-1} \mathcal{V}_{2} E_{2}\left(\lambda_{2}\right) \mathcal{V}_{2}^{-1} \mathcal{V}_{1}=U E(\lambda) U^{-1}$ in $\pi_{1}(\mathfrak{A})=C_{r}^{*}(\Gamma, \bar{\sigma}) \otimes \mathcal{K}\left(L^{2}\left(\mathbb{R}^{n}\right)^{N}\right)$.

\section{REFERENCES}

[1] A. Figotin, P. Kuchment, Band-Gap Structure of Spectra of Periodic Dielectric and Acoustic Media. I. Scalar model. SIAM J. Appl. Math. 56 (1996), 68-88. II. Two-dimensional photonic crystals. SIAM J. Appl. Math. 56 (1996), 1561-1620.

[2] A. Figotin, P. Kuchment, Spectral properties of classical waves in high-contrast periodic media, SIAM J. Appl. Math. 58 (1998), 683-702.

[3] L. Friedlander, On the density of states of periodic media in the large coupling limit, Commun. Partial Differ. Equations 27 (2002), 355-380.

[4] B. Helffer, A. Mohamed, Caractérisation du spectre essential de l'opérateur de Schrödinger avec un champ magnétique, Ann. Inst. Fourier Grenoble 38 (1988), 95-112.

[5] B. Helffer, A. Mohamed, Semiclassical analysis for the ground state energy of a Schrödinger operator with magnetic wells, J. Funct. Anal. 138 (1996), 40-81.

[6] B. Helffer, A. Morame, Magnetic bottles in connection with superconductivity, J. Funct. Anal. 185 (2001), 604-680.

[7] B. Helffer, A. Morame, Magnetic bottles for the Neumann problem: the case of dimension 3, Spectral and inverse spectral theory (Goa, 2000) Proc. Indian Acad. Sci. (Math. Sci.) 112 (2002), 71-84.

[8] B. Helffer, A. Morame, Magnetic bottles for the Neumann problem: Curvature effects in the case of dimension 3, Preprint mp_arc 01-362 (2001). To appear in Annales de l'ENS 2004.

[9] B. Helffer, J. Nourrigat, Hypoellipticité maximale pour des opérateurs polynômes de champs de vecteurs Birkhäuser, Boston, 1985.

[10] R. Hempel, I. Herbst, Strong magnetic fields, Dirichlet boundaries, and spectral gaps, Commun. Math. Phys. 169 (1995), 237-259.

[11] R. Hempel, K. Lienau, Spectral properties of periodic media in the large coupling limit, Commun. Partial Differ. Equations 25 (2000), 1445-1470.

[12] R. Hempel, O. Post, Spectral gaps for periodic elliptic operators with high contrast: an overview; to appear in Conference proceedings of the ISAAC Conference, Berlin, 2001, preprint math-ph/0207020.

[13] I. Herbst, S. Nakamura, Schrödinger operators with strong magnetic fields: Quasi-periodicity of spectral orbits and topology. In: Differential operators and spectral theory, Amer. Math. Soc. Transl. Ser. 2, v. 189, Amer. Math. Soc., Providence, RI, 1999, pp. $105-123$.

[14] Yu. A. Kordyukov, V. Mathai, M. Shubin, Equivalence of projections in semiclassical limit and a vanishing theorem for higher traces in $K$-theory, preprint math.DG/0305189

[15] V. Mathai, M. Shubin, Semiclassical asymptotics and gaps in the spectra of magnetic Schrödinger operators, Geometriae Dedicata 91(2002), 155-173.

[16] M. Shubin, Discrete magnetic Laplacian, Commun. Math. Phys. 164 (1994), 259-275.

Institute of Mathematics, Russian Academy of Sciences, Ufa, Russia

E-mail address: yuri@imat.rb.ru 